\title{
¿Es segura la cirugía ambulatoria de reconstrucción de ligamento cruzado anterior? Experiencia a dos años de seguimiento clínico
}

\section{Is Outpatient Reconstruction of the Anterior Cruciate Ligament a Safe Procedure? Experience with Two Years of Follow-up}

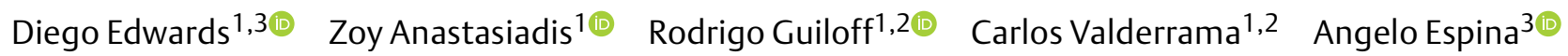

Sergio Arellano ${ }^{1}$ Andrés Schmidt-Hebbel ${ }^{10}$ Alex Vaisman ${ }^{10}$

${ }^{1}$ Clínica Alemana, Santiago, Región Metropolitana, Chile

2 Facultad de Medicina, Universidad del Desarrollo, Santiago, Chile

${ }^{3}$ Departamento de Ortopedia y Traumatología, Hospital Padre

Hurtado, Santiago, Región Metropolitana, Chile

Rev Chil Ortop Traumatol 2021;62(2):e118-e126.
Address for correspondence Diego Edwards, MD, Clinica Alemana, Avenida Vitacura 5951, 7630000, Vitacura, Región Metropolitana, Chile (e-mail: edwardsdiego@gmail.com).

\section{Resumen \\ Palabras Clave \\ - ligamento cruzado anterior \\ - reconstrucción \\ - ambulatorio \\ - complicaciones \\ - costos}

Introducción La cirugía ambulatoria permite una reducción importante del costo en procedimientos de alta prevalencia; no obstante, siempre debe resguardarse la seguridad del paciente.

Objetivo Evaluar las complicaciones operatorias tempranas y resultados funcionales en pacientes sometidos a reconstrucción de ligamento cruzado anterior (R-LCA) en cirugía ambulatoria. Se analiza además una estimación en la reducción de costos por programa ambulatorio.

Material y Métodos Estudio retrospectivo de pacientes sometidos a R-LCA con técnica hueso-tendón-hueso $(\mathrm{HTH})$ en pabellón ambulatorio en un mismo centro, entre 2016 y 2018. Se excluyeron pacientes con menos de un año de seguimiento. Se utilizó el mismo protocolo anestésico: anestesia espinal y bloqueo sensitivo único, asociado a analgesia postoperatoria por vía oral. Se entregó a pacientes instructivo de cuidados postoperatorios, síntomas de alarma, y ejercicios de fisioterapia al alta. Se realizó encuesta telefónica al tercer día para evaluar el estado general y las complicaciones, $y$, al final del seguimiento, para evaluación funcional mediante las escalas de Tegner y Lysholm pre- y postquirúrgicos. Se identificaronó a pacientes no dados de alta el mismo día, consulta precoz no programada, y reintervenciones. Se realizó un análisis de costo para evaluar el ahorro por procedimiento ambulatorio versus hospitalizado.

Resultados Se operaron 36 pacientes de forma ambulatoria. En $4(11,1 \%)$ se asoció a sutura meniscal con técnica dentro-fuera. recibido

05 de mes de julio de 2020

aceptado

12 de marcha de 2021
DOI https://doi.org/

$10.1055 / \mathrm{s}-0041-1735548$. ISSN $0716-4548$. (c) 2021. Sociedad Chilena de Ortopedia y Traumatologia. All rights reserved.

This is an open access article published by Thieme under the terms of the Creative Commons Attribution-NonDerivative-NonCommercial-License, permitting copying and reproduction so long as the original work is given appropriate credit. Contents may not be used for commercial purposes, or adapted, remixed, transformed or built upon. (https://creativecommons.org/ licenses/by-nc-nd/4.0/)

Thieme Revinter Publicações Ltda., Rua do Matoso 170, Rio de Janeiro, RJ, CEP 20270-135, Brazil 
La encuesta postoperatoria inicial fue respondida por 23 pacientes $(63,8 \%)$; todos presentaron buen estado general: $43 \%$ sin dolor y $57 \%$ con molestias tolerables. No hubo sangrados.

La encuesta al final del seguimiento (promedio: $22,5 \pm 7,9$ meses) fue respondida por 20 pacientes $(55,5 \%)$ : la puntación en las escalas de Tegner y Lysholm aumentó significativamente, de 3 (rango: 1 a 6$)$ a 6 (rango: 3 a 8$)(p=0,0001)$ y de 44 (rango: 12 a 81$)$ a 91 (rango: 61 a 100) $(p=0,0001)$, respectivamente.

Todos fueron dados de alta el mismo día de la operación.

Hubo 2 (5,5\%) consultas precoces, una por caída en domicilio con dehiscencia de herida operatoria, y otra por hematoma no complicado. Se registraron dos reintervenciones: una dehiscencia de herida operatoria y una fractura de patela.

La reducción de costos por realizar el procedimiento de forma ambulatoria fue de 203.205 pesos/paciente.

Conclusión La cirugía ambulatoria de R-LCA mediante la técnica HTH fue un procedimiento seguro en esta serie, con un manejo adecuado del dolor y resultados funcionales satisfactorios al mediano plazo. Se asoció además a una reducción en estimación de costos.

\section{Abstract}

\section{Keywords}

- anterior cruciate ligament

- reconstruction

- outpatient

- complications

- costs

Introduction Outpatient procedures allow for an important cost reduction in highprevalence procedures; however, patient safety must always be ensured.

Objective To evaluate the early complications and functional scores of patients undergoing an anterior cruciate ligament reconstruction (ACLR) as an outpatient procedure. An estimated cost reduction is also analyzed.

Materials and Methods A retrospective study of patients undergoing outpatient ACLR with a bone-patellar tendon-bone (BPTB) technique in one center between 2016 and 2018. Patients with less than one year of follow-up were excluded. All patients were submitted to the same anesthetic protocol: spinal anesthesia, a one-shot echo-guided adductor canal nerve block, and outpatient analgesics. Upon discharge, all patients received instructions regarding postoperative care, physical therapy exercises, and red flags. A telephone survey was conducted on the third day to evaluate the general conditions and complications, as well at the final follow-up, to collect pre- and postoperative Tegner and Lysholm functional scores. Patients who were not discharged on the same day, early non-scheduled visits, and re-interventions were recorded.

A cost-reduction analysis was performed for the inpatient versus outpatient procedures. Results In total, 36 patients were submitted to an outpatient procedure, and 4 $(11.1 \%)$ had an outside-in meniscal suture.

The survey was filled out by 23 patients (63.8\%); all were in good general condition: $43 \%$ reported no pain and 57\%, tolerable pain. No bleeding was observed.

The survey at the end of the follow-up (average: $22.5 \pm 7.9$ months) was filled out by 20 patients (55.5\%); the scores on the Tegner and Lysholm scales improved significantly, from 3 (range: 1 to 6$)$ to 6 (range: 3 to 8$)(p=0.0001$ ), and from 44 (range: 12 to 81 ) to 91 (61 to 100$)(p=0.0001)$ respectively.

All patients were discharged on the same day of surgery.

There were 2 (5.5\%) early visits, one due to a fall at home withdehiscence of the surgical wound, and one due to a non-complicated hematoma. Two re-interventions at the end of the follow-up were recorded: traumatic surgical-wound dehiscence and a patellar fracture. The cost reduction for the outpatient procedure was of 203, 205 pesos per patient Conclusion The outpatient ACLR with the BPTB technique was a safe procedure in the present series, with adequate pain management and satisfactory functional scores at the medium-term follow-up. It was also associated with a reduction in cost estimates. 


\section{Introducción}

La cirugía de reconstrucción de ligamento cruzado anterior (R-LCA) es una de las más prevalentes en el campo de la ortopedia, la que ha tenido un aumento importante en su número las últimas décadas. Sólo en Estados Unidos, aumentó de 86.687 cirugías/año, en 1994, a 129.836, en 2006. ${ }^{1}$ En Francia, el año 2013 se realizaron 41.937 RLCAs, ${ }^{2}$ y en Australia, más de $10 \mathrm{mil} / \mathrm{año}$, con un aumento del $14 \%$ por cada año entre 2003 y $2008 .^{3}$ Esta cirugía clásicamente se ha llevado a cabo de forma hospitalizada; ${ }^{4}$ sin embargo, en la última década, con el fin de disminuir los costos asociados al procedimiento, se ha comenzado a realizar de manera más frecuente de forma ambulatoria.

Esta modalidad se ha vuelto más popular en países como Estados Unidos, donde se ha publicado que entres los años 1997 y 2006 se aumentó la tasa de $57,3 \%$ a $95,1 \%,{ }^{5}$ y aumentaron los casos ambulatorios entre los años 1994 y 2007 un 300\%. ${ }^{6}$ Una tendencia similar se ha visto en países del norte de Europa, como es el caso del $79 \%$ de procedimientos ambulatorios que presenta Dinamarca; ${ }^{7}$ sin embargo, no ocurre lo mismo en otros países, como Inglaterra, ${ }^{8}$ Alemania, Austria, y Brasil. ${ }^{9}{ }^{90}$ En el caso de Francia, de las casi 42 mil cirugías realizadas el año 2013, sólo un $3 \%$ fue hecho de forma ambulatoria, ${ }^{11}$ mientras que la mediana de estadía hospitalaria fue de 3 a 5,5 días. ${ }^{12}$

En cuanto a los costos, la literatura ${ }^{13-15}$ demuestra un menor gasto importante a la hora de comparar ambas modalidades en la R-LCA, con ahorros que pueden ir de 1.371 hasta los 7.390 dólares por cada paciente.

En Chile, no contamos actualmente con datos publicados respecto a programas ambulatorios en la R-LCA y su eficacia, lo que podría tener un impacto económico muy relevante. La hipótesis de nuestro estudio es que la R-LCA llevada a cabo de forma ambulatoria presenta una baja tasa de complicaciones operatorias tempranas, con una mejoría de lapuntuación en las escalas clínicas.

Por lo tanto, este estudio tiene como objetivo evaluar las complicaciones operatorias tempranas y sus resultados funcionales. Como objetivo secundario, se analizó una estimación de la reducción de costos en pacientes sometidos a R-LCA mediante la modalidad de cirugía ambulatoria.

\section{Material y Métodos}

Se realizó un estudio retrospectivo de pacientes sometidos a R-LCA con técnica hueso-tendón-hueso (HTH) en pabellón ambulatorio de un mismo centro, entre 2016 y 2018. Se excluyeron pacientes con menos de un año de seguimiento. Se utilizó el mismo protocolo anestésico: anestesia espinal y bloqueo sensitivo único asociado a analgesia postoperatoria con paracetamol y antiinflamatorios no esteroides (AINEs) a cada hora por vía oral, más tramadol 15 gotas de rescate. Se entregó a todos los pacientes un instructivo de cuidados postoperatorios, síntomas de alarma, y ejercicios al alta. Al tercer, día se realizó una encuesta telefónica por una enfermera del servicio ambulatorio, para evaluar el estado general, el dolor, y las complicaciones. Al final del seguimiento, se realizó una evaluación funcional pre y postquirúrgica mediante las escalas de Tegner y Lysholm. Mediante el registro clínico, se identificaron a pacientes a los que no se haya podido dar de alta el mismo día de la cirugía, y aquellos casos de consulta precoz no programada o reintervenciones.

El estudio contó con la aprobación del comité de ética de la institución.

\section{Diseño del Estudio}

Este estudio corresponde a un análisis retrospectivo de una casuística de pacientes operados por lesión de ligamento cruzado anterior mediante reconstrucción en unidad de cirugía ambulatoria de un mismo hospital público de Santiago, durante los años 2016 a 2018. Los pacientes fueron entrevistados mínimo dos días previo a la cirugía por enfermera de pabellón, para conocer su estado actual general y sus antecedentes, además de entregar indicaciones para el día de su cirugía. Todos los pacientes fueron operados con la misma técnica quirúrgica y el mismo protocolo anestésico, y dados de alta el mismo día, entre 2 a 4 horas luego de la operación, teniendo un dolor en la escala visual analógica (EVA) $\leq 3$ y logrando bipedestar con ayuda de bastones. De forma prospectiva, se les contactó para completar la encuesta telefónica en el postoperatorio temprano y luego de forma retrospectiva para completar las encuestas de estudio funcional alejadas.

\section{Criterios de Selección}

Se incluyeron a pacientes:

- Con lesión de ligamento cruzado anterior (con o sin lesión meniscal y/o condral);

- Mayores de 15 años;

- Con puntación 1 ó 2 en la escala de la Sociedad Americana de Anestesiólogos (American Society of Anaesthesiologists, ASA, en inglés);

- Del mismo centro y operados por el mismo equipo quirúrgico;

- Operados con la técnica de HTH;

- Con los mismos protocolos anestésico y analgésico perioperatorios; $\mathrm{y}$

- Con seguimiento mínimo de 12 meses al momento del estudio.

Se excluyeron a pacientes:

- Con lesiones multiligamentosas;

- Con registro incompleto de ficha clínica;

- Sometidos a otras técnicas de reconstrucción; y

- Con imposibilidad de realizar cirugía ambulatoria por condición médica.

\section{Protocolos Analgésico y Anestésico Perioperatorios}

Todos los pacientes fueron premedicados 2 horas antes de su cirugía con paracetamol $1 \mathrm{~g}$ y pregabalina $75 \mathrm{mg}$ por vía oral. El procedimiento anestésico consistió en una anestesia espinal con bupivacaína al 0,75\% (11,25 mg) más fentanil (15 mcg), y, luego de la cirugía, se realizó un bloqueo 

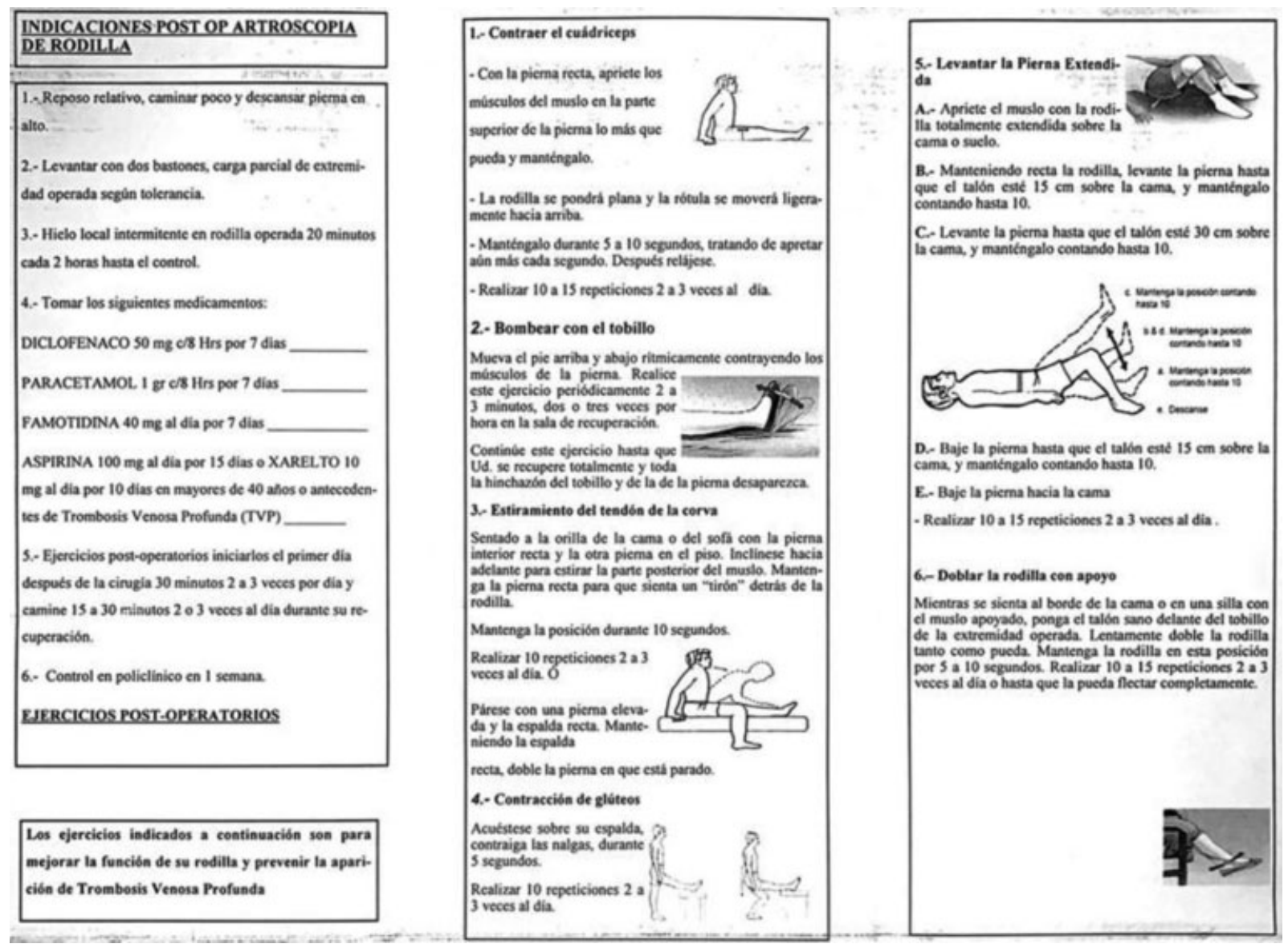

Fig. 1 Instructivo para pacientes en domicilio.

sensitivo único del canal de los aductores con bupivacaína al 0,33\% (30 mL) mediante apoyo ecográfico.

En el postoperatorio temprano, se indicaron antiinflamatorios según receta (diclofenaco $50 \mathrm{mg}$ ) asociado a paracetamol $1 \mathrm{~g}$, ambos por vía oral, cada 8 horas y por 7 días. Se agregó además pregabalina $150 \mathrm{mg}$ cada noche las primeras 2 noches, y se indicó tramadol 15 gotas de rescate. Se agregaron en algunos casos famotidina para protección gástrica, y tromboprofilaxis según la puntación de Caprini.

Finalmente, se les entregó un instructivo con los síntomas de alarma para consultar en la urgencia y una pauta de ejercicios para realizar en su domicilio (-Figura $\mathbf{1}$ ).

\section{Técnica Quirúrgica}

Todos los pacientes fueron operados de forma artroscópica, con uso de mango de isquemia y mediante la técnica de $\mathrm{HTH}$, empezando siempre con un examen físico bajo anestesia. Se realizó la toma del injerto con técnica clásica, y se preparó en mesa ad hoc logrando diámetro de $9 \mathrm{~mm}$ a $10 \mathrm{~mm}$. Bajo visión artroscópica, se evaluaron posibles lesiones asociadas (condrales y meniscales), y se trataron según criterio del primer cirujano (condroplastía/microfractura condral, resección/sutura meniscal). Se crearon los túneles en zona de huella anatómica mediante técnica de portal anteromedial, comenzando por túnel femoral en flexión de $120^{\circ}$, y, luego, el túnel tibial con uso de guía. Se fijó el injerto en $20^{\circ}$ de flexión con uso de tornillos interferenciales de titanio (Arthrex Corporation, Naples, FL, EEUU), logrando restaurar la estabilidad de la rodilla, sin necesidad de realizar un refuerzo anterolateral.

No se utilizó drenaje en ninguno de los casos. Se cubrió la zona operatoria con vendaje tipo Robert-Jones, sin uso de inmovilizador.

\section{Seguimiento Ambulatorio}

Se realizó encuesta telefónica al tercer día para evaluar el estado general, el dolor, y las complicaciones, y, al final del seguimiento, para evaluación funcional mediante las escalas de Tegner y Lysholm pre y postquirúrgicas. Se identificaron consulta precoz no programada y reintervenciones.

Los pacientes fueron contactados telefónicamente por enfermera del pabellón al tercer día para llenar la encuesta ambulatoria ad hoc (-Figura 2), y se evaluaron parámetros generales, como el estado general, el dolor, y las complicaciones que puedan haber presentado; estas respuestas son cuantificadas mediante una escala, lo que permite un registro informático y la toma de decisiones (-Figura 3). Además, se averiguaron posibles consultas precoces al servicio de urgencias. Al momento del estudio, se volvió a llamar por teléfono para realizar encuestas funcionales alejadas, basadas en las escalas de Tegner y Lysholm, para el pre y postquirúrgico. 
122 Reconstrucción ambulatoria de ligamento cruzado anterior Edwards et al.

VARIABLES DEL CONTROL TELEFÓNICO

\begin{tabular}{|c|c|c|}
\hline \multirow{4}{*}{$\begin{array}{l}\text { ESTADO GENERAL } \\
\text { EG: estado del paciente en el momento } \\
\text { de la llamada }\end{array}$} & 2 & $\begin{array}{l}\text { Tranquilo y/o eufórico. Plena satisfacción de su bienestar. Ritmo urinario normal. } \\
\text { Sueño normal }\end{array}$ \\
\hline & 1 & $\begin{array}{l}\text { Preocupado por su estado. No muy satisfecho. Cefalea leve. Ha dormido poco pero } \\
\text { suficiente. Ritmo de diuresis normal }\end{array}$ \\
\hline & 0 & $\begin{array}{l}\text { Ansiedad moderada. Mareado o inquieto. Cefalea intensa. Nada satisfecho. No ha } \\
\text { podido descansar. Le cuesta orinar/no tiene ritmo urinario habitual. Estreñido } 0 \\
\text { con diarrea }\end{array}$ \\
\hline & -8 & $\begin{array}{l}\text { Disnea, estupor o palidez con sensación de gravedad. No ha podido dormir. No ha } \\
\text { orinado y tiene sensación de malestar intenso por globo vesical }\end{array}$ \\
\hline \multirow{4}{*}{$\begin{array}{l}\text { DOLOR } \\
\text { D: intensidad del dolor en el momento } \\
\text { de la llamada }\end{array}$} & 2 & Sin dolor o dolor leve, tanto en reposo como en movimiento \\
\hline & 1 & Dolor aceptable que no impide ni el movimiento ni dormir \\
\hline & 0 & $\begin{array}{l}\text { Dolor moderado, aceptable en reposo, pero importante en el movimiento, altera } \\
\text { ritmo de sueño }\end{array}$ \\
\hline & -8 & $\begin{array}{l}\text { Dolor intenso incluso en reposo con el tratamiento analgésico correctamente } \\
\text { administrado, acompañado por palidez, sudoración. Bradicardia o taquicardia, } \\
\text { hipo-hipertensión }\end{array}$ \\
\hline \multirow{4}{*}{$\begin{array}{l}\text { TOLERANCIA } \\
\text { T: normalidad de la dieta que realiza el } \\
\text { paciente }\end{array}$} & 2 & Sin restricciones en la dieta \\
\hline & 1 & La dieta viene determinada por la cirugía \\
\hline & 0 & $\begin{array}{l}\text { Malestar, náuseas o vómitos, tras un periodo prudencial de dieta absoluta. Se } \\
\text { incumple dieta propuesta al alta }\end{array}$ \\
\hline & -8 & $\begin{array}{l}\text { Vómitos incoercibles incluso después de una dieta absoluta. No es posible la } \\
\text { rehidratación oral }\end{array}$ \\
\hline \multirow{2}{*}{ SANGRADO } & 2 & No hay sangrado \\
\hline & 1 & Sangrado normal en cantidad y ritmo, según la intervención \\
\hline \multirow{2}{*}{$\begin{array}{l}\text { S: intensidad del sangrado durante el } \\
\text { postoperatorio }\end{array}$} & 0 & Crece el manchado del apósito a lo largo de la tarde \\
\hline & -8 & La herida sangra de forma copiosa, empapa y desborda el apósito \\
\hline \multirow{2}{*}{ FIEBRE } & 0 & Temperatura corporal inferior a $38^{\circ} \mathrm{C}$ \\
\hline & -8 & Temperatura corporal superior a $38^{\circ} \mathrm{C}$ \\
\hline \multirow[b]{2}{*}{ ANOMALIA HERIDA } & 0 & Normalidad en la herida \\
\hline & -8 & $\begin{array}{l}\text { Cambios isquémicos en el miembro intervenido, apertura brusca de los puntos } \\
\text { o presencia de cualquier cambio que parezca que precise asistencia médica } \\
\text { inmediata }\end{array}$ \\
\hline \multirow{2}{*}{ CUMPLIMIENTO DEL TRATAMIENTO } & 0 & Cumplimiento del tratamiento y de las dietas \\
\hline & -2 & Incumplimiento del tratamiento o de las medidas higiénicas \\
\hline
\end{tabular}

Fig. 2 Encuesta telefónica temprana.

Se consignaron las complicaciones: pacientes que no se hayan podido dar de alta el mismo día; consultas precoces en el servicio de urgencias (menos de 7 días postoperado); y la presencia de reintervenciones, mediante la revisión de ficha electrónica y de protocolos quirúrgicos del hospital.

- 8-4: evolución dentro de los parámetros normales.

- 3-0: es necesaria una nueva llamada en una hora hasta detectar la mejoria del paciente.

- Negativo: el paciente necesita asistencia en el domicilio o tiene que desplazarse al hospital.

Fig. 3 Puntuación de la encuesta telefónica.

\section{Análisis de Costos}

Se consignaron los valores de una hospitalización habitual por una noche en el hospital en cama básica, considerando el costo del día/cama, medicamentos endovenosos, atención kinésica y evaluación médica al día siguiente, según los valores entregados por el Departamento de Finanzas de la institución.

\section{Análisis Estadístico}

Se compararon las diferencias entre las evaluaciones pre y postoperatorias. Se calcularon para las escalas funcionales (Tegner y Lysholm) la diferencia de los resultados obtenidos para pre y postoperatorio; se verificó la distribución de los datos a través del test de Shapiro-Wilks, y se utilizó la prueba $t$ de Student en muestras pareadas para aquellas evaluaciones con distribución normal, y el test de 
Wilcoxon-Rank para aquellas no paramétricas. Se estableció una significancia estadística con $p<0,05$.

\section{Resultados}

Los pacientes operados de forma ambulatoria para este período fueron 36, de los cuales $23(63,8 \%)$ completaron la encuesta temprana, y 20 (55,5\%), la encuesta alejada (escalas funcionales). Todos los pacientes pudieron ser dados de alta el mismo día, y asistieron a sus controles médicos durante el primer mes.

El 33\% y el 69\% presentaron lesiones condrales y meniscales asociadas respectivamente. En 4 casos (11,1\%), se realizó una sutura meniscal, todas con técnica dentro-fuera.

Respecto a la encuesta del tercer día postoperatorio (encuesta temprana), de los $23(63,8 \%)$ pacientes que contestaron la encuesta, todos presentaron un buen estado general: un $43 \%$ manifestó no tener dolor, y un $57 \%$, tener sólo molestias leves que no impedían el movimiento ni dormir. Ningún paciente reportó presentar un sangrado de la herida quirúrgica.

Respecto a las escalas funcionales al final del seguimiento (encuesta alejada), el resultado en la escala de Tegner tuvo un aumento significativo de nivel, de 3 (rango: 1 a 6 ) a 6 (rango: 3 a 8 ) puntos ( $p=0,0001)$, y el de la escala de Lysholm, de 44 (rango: 12 a 81 ) a 91 (rango: 61 a 100$)$ puntos $(p=0,0001$ ) ( - Figura 4). El tiempo de seguimiento promedio al momento de hacer la encuesta fue de $22,5 \pm 7,9$ meses.

\section{Complicaciones}

Ningún paciente debió quedar hospitalizado luego de la cirugía (todos con dolor tolerable y sin sangrado de la herida).
Respecto a las consultas precoces en el servicio de urgencias (primera semana postoperatoria), se identificaron 2 casos (5,5\%):

- Paciente con caída a nivel en su primer día postoperatorio al levantarse sin bastones, sufriendo un golpe directo en su rodilla operada y una consecuente dehiscencia completa de la herida quirúrgica; el paciente debió ser intervenido de forma urgente para aseo y nuevo cierre de la herida por el traumatólogo de urgencia;

- Segundo paciente acude al servicio de urgencias al quinto día de evolución preocupado por hematoma en zona operatoria (en relación al uso de rivaroxaban por tromboprofilaxis). No presentaba complicación; sin sangrado en herida quirúrgica, se envió de vuelta a domicilio sin tratamiento específico.

Se identificaron dos 2 con reintervenciones (5,5\%), dentro de los cuales se encuentra el paciente con dehiscencia traumática de la herida operatoria. El segundo caso corresponde a un paciente con caída a nivel en el suelo mojado del baño de su casa a los 12 días de evolución, con un golpe directo en la rodilla operada; se diagnosticó una fractura de rótula desplazada, la que se operó por el mismo equipo quirúrgico con una reducción y osteosíntesis (-Figura 5). No se presentaron reintervenciones por fallo de la reconstrucción hasta el final del seguimiento.

Respecto a los costos, a través del Departamento de Finanzas del hospital, se obtuvo el valor de todo lo que no se utiliza en un paciente operado de forma ambulatoria; por lo tanto, se consideró el costo del día/cama básica, medicamentos endovenosos, atención kinésica y evaluación médica al día siguiente, lo que dio un total de 203.205 pesos por paciente.

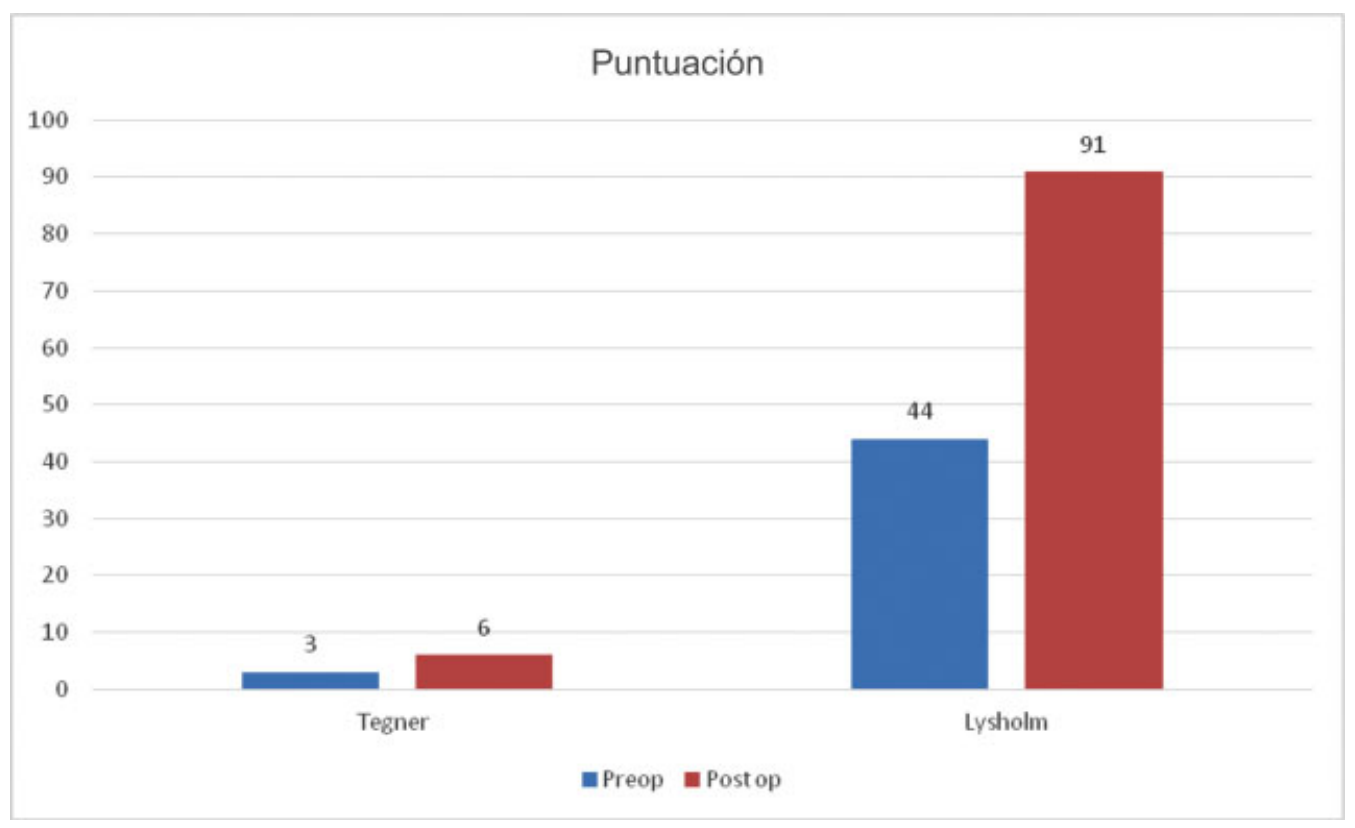

Fig. 4 Resultados funcionales al final del seguimiento (22,5 meses). 


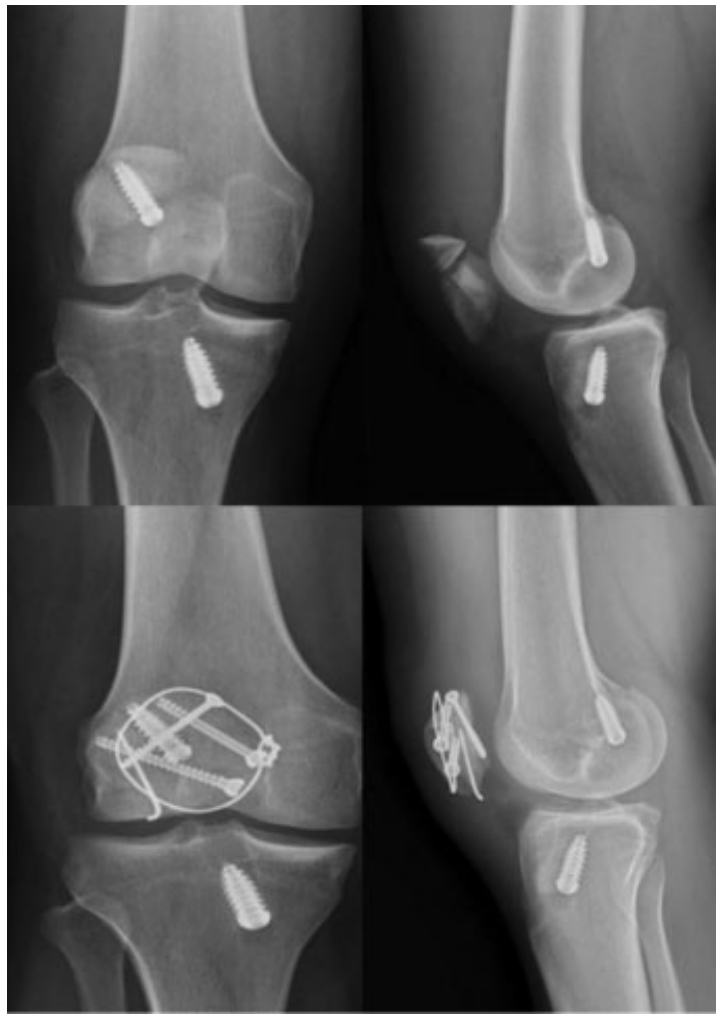

Fig. 5 Fractura de rótula operada con tornillos más obenque circular.

\section{Discusión}

En Chile, no contamos con literatura publicada que evalúe programas ambulatorios en R-LCA, a diferencia de otros países que lo han desarrollado desde hace algunas décadas. $^{1,6}$ Este estudio es el primer reporte nacional respecto a los resultados y complicaciones de cirugía ambulatoria en R-LCA.

Las potenciales desventajas en una R-LCA realizada en forma ambulatoria son la posibilidad de presentar un mal manejo del dolor y complicaciones tempranas en el domicilio, lo que podría generar una alta tasa de readmisión temprana y peores resultados funcionales. En nuestra serie, los pacientes refirieron estar sin dolor o con dolor leve al tercer día, y sin registro de reconsulta por esta causa. Esto concuerda con lo publicado durante la última década, en que se demuestra que es posible lograr un adecuado manejo analgésico en los pacientes operados por R-LCA de forma ambulatoria. Al respecto, destacan trabajos como los de Talwalkar et al., ${ }^{16}$ en el que sólo 2 de los 51 pacientes no pudieron ser dados de alta el mismo día de la cirugía, aunque la razón de esto no fue un mal manejo del dolor, sino que un drenaje con un débito alto. De Beule et al., ${ }^{17}$ en su cohorte de 355 pacientes, publicaron una readmisión durante el primer mes postoperatorio de $1,4 \%$, y otros trabajos, como los de Tierney et al. ${ }^{4}$ (227 R-LCAs), Williams et al. ${ }^{18}$ (50 R-LCAs), y Lunebourg et al. ${ }^{19}$ (30 RLCAs), no reportaron ninguna readmisión en el postoperatorio en relación a la cirugía. Los últimos autores, además, en su estudio compararon la satisfacción de 30 pacientes luego de $\mathrm{u} 1 \mathrm{n}$ mes postoperatorio de forma ambulatoria con 30 pacientes operados de forma hospitalizada, y tuvieron mejores resultados en el grupo ambulatorio.

Para lograr un exitoso manejo del dolor y evitar las reconsultas por esta causa, creemos que es fundamental un adecuado protocolo anestésico. En la literatura, destacan diversos protocolos, como anestesia general (Lefevre et al. ${ }^{20}$ ), anestesia espinal, a veces con infiltración local en la zona de toma del injerto (Talwalkar et al. ${ }^{16}$ ) y/o de las incisiones quirúrgicas (Tierney et al.; ${ }^{2}$ Williams et al. ${ }^{18}$ ), bloqueos de nervio femoral o canal de aductores (Lunebourg et al. ${ }^{19}$ ), o incluso utilizando distintos protocolos en una misma cohorte. Esta falta de uniformidad hace más difícil comparar los resultados entre ellos, pero demuestra que es posible lograr un buen resultado mediante múltiples esquemas. En nuestro estudio, describimos el protocolo utilizado, que consiste en la administración de premedicación oral, anestesia espinal más un bloqueo sensitivo único del canal de los aductores con apoyo ecográfico al finalizar la cirugía, además de un manejo analgésico ad hoc en domicilio. Este protocolo fue convenido con el equipo de anestesistas en base a lo publicado y a la experiencia del equipo tratante. Tal como describimos, mediante esta técnica se logró no tener ninguna consulta temprana no programada por mal manejo del dolor.

Nuestra casuística presenta 2 casos de reconsulta (5,5\%) y 2 reintervenciones $(5,5 \%)$ dentro de los primeros 30 días. La dehiscencia de la herida quirúrgica que requirió aseo y sutura en un paciente fue considerada una complicación temprana; si bien estaba con un bloqueo sensitivo regional, pudiese haber tenido aún algún efecto de bloqueo motor secundario al procedimiento anestésico o de medicamentos. El otro paciente que requirió una reintervención, debido a una fractura de rótula en el mismo lado del procedimiento, presentó una caída por descuido a los 12 días de postoperatorio, cuando ya no estaba recibiendo analgesia de ningún tipo. La tasa de complicaciones asociadas a R-LCA es variable, describiendo incluso hasta un $9 \%,{ }^{1}$ sin que se haya demostrado una tasa mayor asociada al hecho de realizar la cirugía en modalidad ambulatoria. Andrés-Cano et al. ${ }^{21}$ reporta un $13,2 \%$ de reconsultas a urgencias después de $\mathrm{R}$ LCA ambulatorio, en su mayoría por dolor, con un 2,3\% de reingresos asociados a la herida quirúrgica. Liu ${ }^{22}$ reporta un 3,9\% de readmisiones hospitalarias tras R-LCA ambulatoria en el estado de Nueva York, Estados Unidos, destacando que los pacientes operados en hospitales de alto volumen tenían menos probabilidad de reconsultar. Creemos que nuestra tasa de reintervención mayor que lo reportado en la literatura se debe en parte al bajo número de pacientes del estudio (dentro del que se incluye a 1 paciente con una caída a los 12 días, sin relación a un programa hecho de forma ambulatoria), y, al mismo tiempo, al hecho de ser una experiencia inicial, que nos obliga a reforzar la prevención de caídas en el protocolo de manejo de nuestros pacientes.

En relación a los resultados funcionales, algunas de las publicaciones ya mencionadas (Williams et al., ${ }^{18}$ Lunebourg et al., ${ }^{19}$ y Lefevre et al. ${ }^{20}$ ) comparan los resultados entre los 
pacientes ambulatorios y los hospitalizados, sin encontrar diferencias entre los grupos; además, Valkering et al. ${ }^{23}$ publicaron un trabajo en el que comparan de forma prospectiva y randomizada a estos dos grupos, sin encontrar diferencias luego de un año de postoperatorio. Al respecto, si bien no contamos con un grupo control, nuestro estudio demuestra una mejoría en los resultados funcionales con un seguimiento promedio de 22,5 meses, sin reportar ningún caso de fallo o revisión hasta el final del seguimiento.

La realización de la cirugía de R-LCA de forma ambulatoria presenta además muchas otras ventajas, como el menor uso de insumos y recursos en un hospital, disminuyendo considerablemente los costos. La reducción en costos que se logró en este programa de R-LCA ambulatoria correspondió a 203.205 pesos por cada paciente operado. Esta reducción es mucho menor que la reportada en otras series, ${ }^{24}$ lo que se explica por haber sido analizada en un hospital público en Chile donde las prestaciones de salud son de mucho menor costo que en instituciones privadas, donde sólo el valor del día/cama básica puede llegar a más de 600 mil pesos, ${ }^{25,26}$ lo que aumentaría considerablemente el ahorro de realizarse en aquellos establecimientos; sin embargo, en la medida que dicho programa ambulatorio se mantiene en el tiempo, el valor ahorrado logra ser considerable; sólo por los 36 pacientes de nuestra casuística el ahorro económico fue de más de 7 millones de pesos para el sistema de salud. Es importante además resaltar el hecho de que, al no haber usado una cama para el paciente operado, la realización de su cirugía no se condiciona a la disponibilidad de cama, y se logra mantener libre ese cupo para que otro paciente pueda utilizarlo y resolver otro problema en salud, lo que otorga un valor agregado muy relevante y difícil de medir.

Como limitaciones de nuestro estudio, podemos mencionar que presenta un diseño retrospectivo y aún contamos con una casuística pequeña, existiendo un porcentaje alto de pacientes que no cuenta con todas las evaluaciones tempranas y tardías completas. Respecto a la pérdida de seguimiento en la encuesta temprana, esta se debió a pérdida del registro, ya que todos los pacientes fueron debidamente contactados por teléfono al tercer día y evaluados en el control médico temprano, sin reportar otras complicaciones. Desafortunadamente, la falta de disponibilidad de camas en el pabellón central de nuestro centro por alta demanda de otras patologías nos obliga a poder optar, en caso de R-LCA, sólo a la modalidad ambulatoria, por lo que no tenemos un grupo comparativo de manejo hospitalizado.

Como fortalezas, destacamos el hecho de que todos los pacientes en el estudio fueron operados por los mismos equipos quirúrgico y anestésico, lo que hace un trabajo más uniforme, mostrando buenos resultados con un programa ambulatorio que genera ahorros importantes al sistema de salud, y ser los primeros en nuestro país en publicar la experiencia de un programa ambulatorio de RLCA.

\section{Conclusión}

La cirugía ambulatoria de R-LCA mediante la técnica HTH fue un procedimiento seguro en esta serie, con un manejo adecuado del dolor y resultados funcionales satisfactorios al mediano plazo. Se asoció además a una reducción en estimación de costos.

\section{Conflicto de Intereses}

Los autores declaran que no hay conflicto de intereses.

\section{Referencias}

1 Mall NA, Chalmers PN, Moric M, et al. Incidence and trends of anterior cruciate ligament reconstruction in the United States. Am J Sports Med 2014;42(10):2363-2370

2 Baverel L, Demey G, Odri GA, Leroy P, Saffarini M, Dejour D. Do outcomes of outpatient ACL reconstruction vary with graft type? Orthop Traumatol Surg Res 2015;101(07): 803-806

3 Janssen KW, Orchard JW, Driscoll TR, van Mechelen W. High incidence and costs for anterior cruciate ligament reconstructions performed in Australia from 2003-2004 to 2007-2008: time for an anterior cruciate ligament register by Scandinavian model? Scand J Med Sci Sports 2012;22(04):495-501

4 Tierney GS, Wright RW, Smith JP, Fischer DA. Anterior cruciate ligament reconstruction as an outpatient procedure. Am J Sports Med 1995;23(06):755-756

5 Lyman S, Koulouvaris P, Sherman S, Do H, Mandl LA, Marx RG. Epidemiology of anterior cruciate ligament reconstruction: trends, readmissions, and subsequent knee surgery. J Bone Joint Surg Am 2009;91(10):2321-2328

6 Buller LT, Best MJ, Baraga MG, Kaplan LD. Trends in anterior cruciate ligament reconstruction in the United States. Orthop J Sports Med 2014;3(01):2325967114563664

7 Granan L-P, Forssblad M, Lind M, Engebretsen L. The Scandinavian ACL registries 2004-2007: baseline epidemiology. Acta Orthop 2009;80(05):563-567

8 Jameson SS, Dowen D, James P, Serrano-Pedraza I, Reed MR, Deehan D. Complications following anterior cruciate ligament reconstruction in the English NHS. Knee 2012;19(01):14-19

9 Astur DC, Navarro PGR, Fonseca LF, et al. Why do patients undergoing anterior cruciate ligament reconstruction in Brazil stay in hospital for longer periods than in other countries? Prospective evaluation of 30 patients and presentation of possible discharge criteria. Rev Bras Ortop 2013;48(04): 336-340

10 Fischer S, Zechmeister-Koss I. Is day surgery safe? A systematic literature review. Eur Surg -. Acta Chir Austriaca 2014;46:103-112

11 http://www.scansante.fr/applications/statistiques-activiteMCO-par-GHM/submit snatnav $=\&$ snatdoc $=\&$ mbout $=$ \&annee $=2013 \&$ base $=0 \&$ noreg $=99 \& \mathrm{ghm}=08 \mathrm{C} 34 \& \mathrm{ok}=$ Lancer $+\mathrm{le}+$ traitement (consulté en ligne le 12 avril 2016)

12 Hulet C, Pineau V, Burdin G, Delforge S, Klebaner I, Rochcongar G. État deslieux de la chirurgie ambulatoire pour l'orthopédie traumatologie en 2012. Acad Natl Chir 2012;11:36-39

13 Kao JT, Giangarra CE, Singer G, Martin S. A comparison of outpatient and inpatient anterior cruciate ligament reconstruction surgery. Arthroscopy 1995;11(02):151-156

14 Novak PJ, Bach BRJ Jr, Bush-Joseph CA, Badrinath S. Cost containment: a charge comparison of anterior cruciate ligament reconstruction. Arthroscopy 1996;12(02):160-164

15 Malek MM, DeLuca JV, Kunkle KL, Knable KR. Outpatient ACL surgery: a review of safety, practicality, and economy. Instr Course Lect 1996;45:281-286 
16 Talwalkar S, Kambhampati S, De Villiers D, Booth R, Stevenson AL. Day case anterior cruciate ligament reconstruction: a study of 51 consecutive patients. Acta Orthop Belg 2005;71(03):309-314

17 De Beule J, Vandenneucker H, Claes S, Bellemans J. Can anterior cruciate ligament reconstruction be performed routinely in day clinic? Acta Orthop Belg 2014;80(03):391-396

18 Williams JS Jr, Wexler G, Novak PJ, Bush-Joseph CA, Bach BR Jr, Badrinath SK. A prospective study of pain and analgesic use in outpatient endoscopic anterior cruciate ligament reconstruction. Arthroscopy 1998;14(06):613-616

19 Lunebourg A, Ollivier M, Delahaye D, Argenson JA, Parratte S. Better satisfaction of patients operated on anterior cruciate ligament reconstruction in outpatient setting. A prospective comparative monocentric study of 60 cases. Arch Orthop Trauma Surg 2016;136(12):1709-1715

20 Lefevre N, Servien E, Colombet P, et al; French Arthroscopic Society. French prospective multicenter comparative assessment of ambulatory surgery feasibility in anterior cruciate ligament reconstruction. Orthop Traumatol Surg Res 2016;102(8S):S257-S263
21 Andrés-Cano et al. Postoperative complications of anterior cruciate ligament reconstruction after ambulatory surgery. Rev Esp Ortop Traumatol 2015;59(03):157-164

22 Liu et al. Thirty-Day acute health care resource utilization following outpatient anterior cruciate ligament surgery. Reg Anesth Pain Med 2018;43:849-853

23 Valkering KP, van Bergen CJ, Buijze GA, Nagel PH, Tuinebreijer WE, Breederveld RS. Pain experience and functional outcome of inpatient versus outpatient anterior cruciate ligament reconstruction, an equivalence randomized controlled trial with 12 months follow-up. Knee 2015;22(02):111-116

24 Ferrari D, Lopes TJA, França PFA, Azevedo FM, Pappas E. Outpatient versus inpatient anterior cruciate ligament reconstruction: A systematic review with meta-analysis. Knee 2017;24(02):197-206

25 https://www.alemana.cl/wls/arancel/buscarc.htm? idClasificacion $=1$ \& pagina $=1 \&$ cantidad $=100$

26 https://www.clinicalascondes.cl/Dev_CLC/media/Otros/ArancelWEB-PRESTACIONES-CLINICAS-PUBLICAR_2019_25012019.pdf 